Artículo

\title{
Análisis de las publicaciones españolas en la categoría Psychology Educational de la Web of Science durante el periodo 2004-2013
}

\author{
Francisco González Sala ${ }^{\mathrm{a}, *}$ y Julia Osca Lluch ${ }^{\mathrm{b}}$ \\ a Departamento de Psicología Evolutiva y de la Educación, Universidad de Valencia , Valencia, España \\ ${ }^{\mathrm{b}}$ Instituto de Gestión e Innovación del Conocimiento INGENIO, Valencia, España
}

\section{INFORMACIÓN DEL ARTÍ́CULO}

\section{Historia del artículo:}

Recibido el 27 de abril de 2015

Aceptado el 20 de julio de 2015

On-line el 20 de agosto de 2015

\section{Palabras clave:}

Psicología Educativa

Bibliometría

Web of Science

Producción científica española

\begin{abstract}
R E S U M E N
El presente trabajo tiene como objetivo analizar la productividad y la visibilidad de la producción científica española en la Psicología Educativa durante el período 2004-2013, utilizando las técnicas bibliométricas. Se han seleccionado los trabajos publicados en la totalidad de revistas incluidas en la categoría temática Psychology Educational de la Web of Science, durante los años 2004 a 2013, realizados en alguna institución española. Los resultados muestran un aumento de la producción a partir del 2006, siendo los artículos la tipología documental más representada, con 456. Estos son firmados por 1.001 autores, habiendo más mujeres que hombres, si bien son los hombres los que más artículos firman y son los más representados como medianos y grandes productores. Las revistas más utilizadas son Infancia y Aprendizaje y la Revista de Psicodidáctica. La universidad es el sector más representado, siendo los investigadores de 11 de ellas los que firman la mitad de la producción.
\end{abstract}

(c) 2015 Instituto de Ciencias de la Educación de la Universidad de Oviedo. Publicado por Elsevier España, S.L.U. Este es un artículo Open Access bajo la licencia CC BY-NC-ND (http://creativecommons.org/licenses/by-nc-nd/4.0/).

\section{Analysis of Spanish publications in the Educational Psychology category of the Web of Science for the period 2004-2013}

\section{A B S T R A C T}

This paper aims to analyse productivity and visibility of the Spanish scientific production in educational psychology during the period 2004-2013, using bibliometric techniques. The selected papers were all published in journals that included the subject category, Educational Psychology, in the Web of Science, from the years 2004 to 2013, written in a Spanish institution. The results show an increase in production from 2006, with articles being the most representative type of document, with a total of 456 . Of the 1,001 authors, there were more females than males, even though men have the greatest number of signed articles and are the most represented as medium and large producers. The most used journals are Infancia y Aprendizaje and Revista de Psicodidáctica. The university is the sector most represented, and 11 researchers sign half of the total production.

(C) 2015 Instituto de Ciencias de la Educación de la Universidad de Oviedo. Published by Elsevier España, S.L.U. This is an open access article under the CC BY-NC-ND license (http://creativecommons.org/licenses/by-nc-nd/4.0/).

\footnotetext{
* Autor para correspondencia: Dpto. de Psicología Evolutiva y de la Educación. Universidad de Valencia. Avda. Blasco Ibáñez, 21, 46019, Valencia (España). Tel.: +9639838 81 .

Correo electrónico: Francisco.Gonzalez-Sala@uv.es (F. González Sala).
}

\section{Introducción}

La actividad investigadora puede ser medida de forma bastante fidedigna analizando el número de publicaciones (productividad) y la frecuencia con que dichos trabajos son citados (visibilidad) (De Moya et al., 2010). Los indicadores bibliométricos permiten analizar la producción científica no solo de forma cuantitativa, sino también de forma cualitativa, es decir, la repercusión que tiene dicha 
producción (Velasco, Eiros, Pinilla y San Román, 2012). La magnitud de los recursos e inversiones destinados a la investigación exigen disponer de medidas o indicadores que cuantifiquen sus resultados e impacto.

Los indicadores bibliométricos se vienen utilizando desde hace décadas para la planificación y puesta en práctica de todos los aspectos relacionados con la evaluación de la producción y de la productividad científica (Buela-Casal, 2010), ya que permiten caracterizar de forma muy precisa el estado de desarrollo de la investigación y, por tanto, fundamentar la toma de decisiones sobre la política científica (Cunningham, 1997).

El número total de publicaciones científicas y el número total de citas recibidas por esas publicaciones son indicadores bibliométricos cada vez más utilizados de forma conjunta para valorar la cantidad y la calidad de la investigación que se realiza en un país. Los métodos clásicos para valorar la calidad de una publicación incluyen el índice de impacto de la revista (Garfield, 2006) y el puesto de esa revista dentro del grupo de publicaciones de la misma área temática. Otros métodos más novedosos se basan en el número de citas recibidas por las publicaciones y uno de los indicadores de este tipo más utilizados es el índice h (de Hirsch), que consiste en ordenar las publicaciones por el número de citas recibidas, y tomar el número del artículo que recibe al menos el mismo número de citas. El índice h surge del supuesto de que el número de citas que recibe un científico constituye un mejor indicador de la relevancia de su trabajo que el número de artículos que publica o en qué revistas lo hace (Hirsch y Buela-Casal, 2014). Los indicadores bibliométricos también permiten identificar a los autores más productivos, los centros generadores de la investigación, las fuentes primarias en las que se publica los trabajos, la repercusión y el impacto que tienen en los trabajos posteriores y los patrones de colaboración existente entre los autores.

La bibliometría se ha extendido e implantado como técnica metodológica imprescindible para la evaluación de la producción científica y de todos los fenómenos ligados a la comunicación de la ciencia, convirtiéndose en una herramienta fundamental para construir y conocer el presente y la historia de la investigación. Después de la medicina, la psicología es la ciencia que más ha utilizado esta técnica de estudio (Delgado-López-Cozar, Torres-Salinas, Jiménez-Contreras y Ruíz-Pérez, 2006). El interés por la producción de la investigación científica en la psicología española ha venido creciendo en los últimos años dando lugar a multitud de trabajos, algunos de los cuales permiten conocer la evolución de la producción científica española. En este sentido, según un estudio de la Fundación Española para la Ciencia y la Tecnología (2013) a partir del año 2002, la producción científica española muestra un crecimiento acelerado, llegando a duplicarse en el año 2010 con respecto a la producción del año 2001, y se considera que la calidad de la producción científica española va a seguir incrementándose. Las técnicas bibliométricas también se han empleado para el estudio de la productividad científica según el género (Barrios, Villaroya y Borrego, 2013; Bermúdez et al., 2011; Sierra, Buela-Casal, Bermúdez y Pablos-Iglesias, 2009; Torres-Salinas, Muñoz-Muñoz y Jiménez-Contreras, 2011; Velasco, Vilariño, Amado y Fariña, 2014), en el estudio de la composición de los comités editoriales de las revistas científicas (GonzálezSala, Fonseca-Baeza y Osca-Lluch, 2014; Mauleón, Hillán, Moreno, Gómez y Bordons, 2013), en la financiación por comunidades (Buela-Casal et al., 2013), en la calidad de las universidades españolas según su implicación social, labor investigadora y función docente (Beamonte, Casino y Veres, 2013), entre otros. Todo ello ha contribuido a un aumento en el número de tesis doctorales relacionadas directamente con la bibliometría (Osca-Lluch, Haba, Fonseca, Civera y Tortosa, 2013).

El análisis de la especificidad de la participación de las mujeres en la investigación científica forma parte de una problematización de creciente reconocimiento en las agendas de las políticas de ciencia y tecnología en todo el mundo. Visibilizar a las mujeres dando a conocer sus aportaciones en el desarrollo de la ciencia y en el avance del conocimiento sigue siendo una actividad imprescindible en el lento camino hacia la igualdad efectiva entre hombres y mujeres (Osca-Lluch, 2011). En el caso de la Psicología Educativa se observa que existe un aumento de la participación de las mujeres en las revistas de esta área entre 2003 y 2008, si bien este progreso no guarda relación con la ratio de hombres y mujeres en los comités de las organizaciones educativas (Fong, Yoo, Jones, Torres y Decker, 2009). En el caso de la Psicología Educativa Olivas-Ávila, Musi-Lechuga, Guillén-Riquelme y Castro (2012) apuntan que no hay diferencias en función del género en cuanto a la producción de artículos en JCR y en el número de citas recibidas, en cambio sí aparecen diferencias en el número de tesis doctorales dirigidas en esta área, siendo los hombres los que han dirigido un mayor número de tesis.

Evans, Hsieh y Robinson (2005) hablan de un aumento importante de las mujeres como editoras, un ligero incremento en los equipos editoriales de las revistas y de un mantenimiento en el porcentaje de mujeres como autoras en las revistas de Psicología Educativa entre 1996 y 2004. Por su parte, González-Alcaide et al. (2010), Olivas-Avila y Musi-Lechuga (2010) encuentran que en España, en el campo de la psicología en general, la productividad de las mujeres con respecto a los hombres es más baja, situación que también se da en otros países (Amico, Vermigli y Canetto, 2011, Malouff, Schutte y Priest, 2010).

Dada la importancia del análisis de la producción científica a través de indicadores bibliométricos, el presente trabajo tiene como objetivo fundamental analizar la productividad y la visibilidad de la producción científica española en Psicología Educativa durante el período 2004-2013 a partir de los trabajos recogidos en la categoría Psychology Educational de la Web of Science (WoS), utilizando complementariamente, las técnicas bibliométricas y el análisis de redes sociales. Se analiza la evolución temporal de la productividad, los idiomas, los tipos de documentos, las revistas en las que se publica y las características de la producción científica en dichas fuentes, los autores y su colaboración con vistas a conocer las características y la evolución de la Psicología Educativa española en los últimos años.

\section{Método}

\section{Material y procedimiento}

La búsqueda bibliográfica se realizó en la base de datos Social Science Citation Index perteneciente a la WoS de la empresa Thomson Reuters y se llevó a cabo en julio de 2014. Para la realización del estudio se han seleccionado los trabajos publicados en las revistas incluidas en la categoría temática Psychology Educational de la base de datos Journal Citation Report (JCR) entre los años 20042013, realizados en alguna institución española. El estudio de la producción en Psicología Educativa en esta última década obedece a dos criterios, al aumento de la productividad científica española (Fundación Española de Ciencia y Tecnología, 2013) y a la inclusión en los años 2008 y 2009 de dos revistas españolas en el área de Psychology Educational, como fueron Infancia y Aprendizaje y la Revista Psicodidáctica. El número de revistas de psicología incluidas en esta categoría fueron 54.

Con la información bibliográfica y de contenido de los registros recuperados se confeccionó una base de datos relacional. A continuación se efectuó un proceso de homogeneización de las firmas aportadas por los autores en los trabajos, ya que en ocasiones diferentes firmas correspondientes a un mismo autor se presentan desdobladas en dos o más variantes, por varios motivos: porque se 
consigne un solo apellido o los dos, una inicial o las dos en el caso de los nombres compuestos, porque se desarrolle o no el nombre de pila de los autores o por la inclusión de signos gráficos como los guiones para separar los apellidos.

Una vez tratada y homogeneizada la información bibliográfica, se han estudiado los siguientes aspectos: la evolución de la productividad científica y colaboración científica entre los autores, la identificación de los grupos de investigación y un análisis de los hábitos de publicación de los investigadores de esta disciplina.

\section{Variables}

Las variables estudiadas en el presente trabajo han sido: evolución temporal de la producción científica, definida por el número de trabajos recogidos en la WoS desde el 2004 hasta el 2013 en la categoría de Psychology Educational. Tipología documental, que comprende las siguientes categorías -artículos, comunicaciones de congresos, material editorial y revisiones-. Idioma de publicación, se contabiliza el idioma en el que se publica cada trabajo. En cuanto a las características de las revistas se recoge tanto el número de revistas donde han sido publicados los trabajos, como el título, el cuartil referente al año de publicación del trabajo, el idioma y el país de la revista. La variable autores viene definida por el número de autores que han publicado uno o más artículos. Género con las categorías hombre y mujer. La variable productividad o número de firmas por trabajo se ha distribuido en tres niveles del indice de productividad, grandes productores, medianos productores y pequeños productores (Lotka, 1926), resultando que los pequeños productores han firmado un solo trabajo, medianos productores entre 2 y 5 trabajos, y grandes productores seis o más trabajos. La variable institución viene definida por el lugar de trabajo de los autores. En cuanto a la colaboración científica, se diferencia entre las categorías -sin colaboración y con colaboración-, además, se ha realizado una red de colaboraciones entre autores, en la cual los nodos representan los autores y las líneas que unen los diferentes nodos representan las colaboraciones entre ellos. El grosor de los nodos indica el mayor o menor número de trabajos que ha realizado un autor y el grosor de las líneas indica la intensidad de la relación entre los autores.

\section{Análisis}

Se realizaron recuento de frecuencias, cálculo de porcentajes, pruebas $t$ y pruebas $\chi^{2}$ para determinar la existencia de diferencias significativas en las categorías analizadas. Todos estos análisis se llevaron a cabo mediante el paquete estadístico SPSS 20. El análisis de redes de colaboración se realizó mediante el software Pajek y su representación gráfica a través de Ucinet.

\section{Resultados}

\section{Evolución de la producción científica}

Durante el período 2004-2013 se publicaron 509 trabajos realizados por investigadores que trabajan en instituciones españolas, y que fueron incluidos en revistas clasificadas en la categoría Psychology Educational en la WoS. Los trabajos seleccionados incluyeron 1.743 firmas correspondientes a 1.120 autores.

En la figura 1 se muestra la evolución de los trabajos publicados en las revistas de Psicología Educativa incluidas en la base de datos de la WoS durante el período 2004 hasta 2013. Se ha representado en una línea, la producción total de trabajos y en una segunda línea, solamente la evolución de la producción de los artículos. Se puede observar que existe un aumento a lo largo del período estudiado en el número de trabajos y artículos publicados, destacando, sobre todo, el aumento de la producción a partir del año 2006 (43 trabajos)

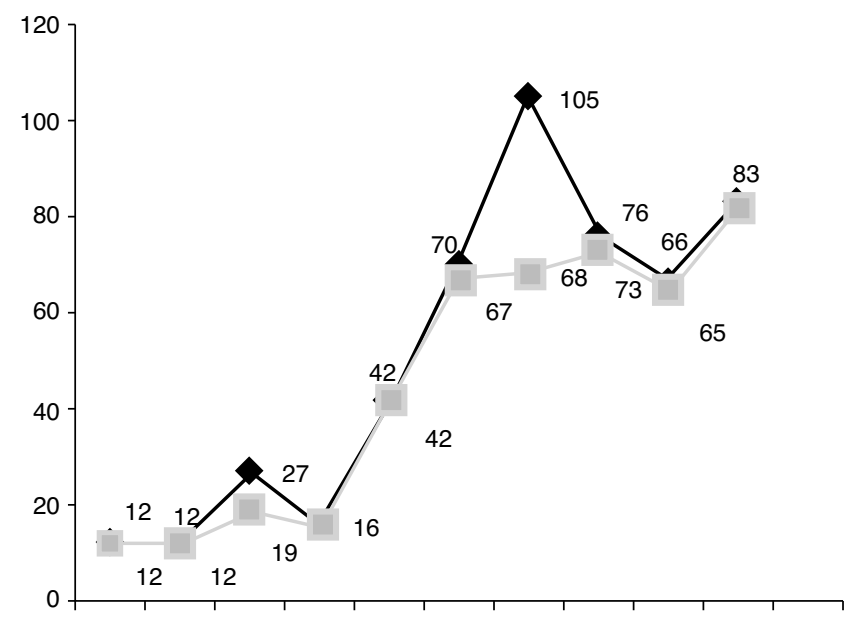

2004200520062007200820092010201120122013

Total producción - Artículos

Figura 1. Evolución del total de trabajos y de artículos en la categoría Psychology Educational de la Web of Science durante el periodo 2004-2013.

con respecto a los años anteriores (12 trabajos en los años 2004 y 2005). Llama la atención el aumento de la producción total de trabajos en el año 2010, sin embargo, se trata de un hecho aislado, como consecuencia de la inclusión en la base de datos de los abstracts de "Third International Conference of IASSID-Europe», publicados en el volumen 23(5) del año 2010 de la revista Journal of Applied Research in Intellectual Disabilities, mientras que el número de artículos se mantiene igual que en el año 2009.

\section{Tipología documental}

La distribución de los trabajos según su tipología documental fue la siguiente, 456 artículos, lo que supone el 89,6\% del total de trabajos, 43 actas de congresos, 7 trabajos referentes a material editorial, 2 revisiones y una revisión de libros. Dada la relevancia que tienen los artículos científicos, y que son los que pueden reflejar mejor la caracterización de la producción científica española en Psicología Educativa, se han seleccionado estos trabajos para la obtención de indicadores sobre la producción científica española en esta disciplina.

\section{Análisis de los autores de artículos científicos y género}

Tras el análisis de los 456 artículos seleccionados se observa que estos incluyeron un total de 1.483 firmas, pertenecientes a un total de 1.001 autores. Al clasificar a los autores según su género, se observa un mayor predominio de mujeres, 547 (54,6\%) frente a $454(45,4 \%)$ hombres.

Por lo que respecta a la producción científica, la gran mayoría de los autores son eventuales, es decir, que han publicado durante todo el período estudiado un solo trabajo ( $\mathrm{n}=728$; 72,7\%), 259 autores $(25,9 \%)$ son medianos productores, ya que firmaron entre 2 y 5 trabajos y solamente 14 autores $(1,4 \%)$ son grandes productores, al firmar 6 o más trabajos. El máximo número de artículos publicados por un mismo autor fue de 12 .

Al analizar la producción de artículos teniendo en cuenta el género, aparecen diferencias estadísticamente significativas $t_{(684,655)}=4,842, \mathrm{p}<0,001$; siendo los hombres los que presentan una mayor producción $(M=1,67, D T=1,33)$ frente a las mujeres $(M=1,33, D T=0,74)$. Como se puede ver en la tabla 1 existe un mayor predominio de hombres como medianos y grandes productores frente a las mujeres, las cuales presentan un mayor porcentaje 
Tabla 1

Distribución de la productividad por género de artículos en la categoría Psychology Educational de la Web of Science durante el periodo 2004-2013

\begin{tabular}{lrrr}
\hline & \multicolumn{2}{c}{ Género } & \\
\cline { 2 - 3 } Productores & Hombre & Mujer & Total \\
\hline Grandes & 12 & 2 & \\
N & $85,7 \%$ & $14,3 \%$ & 14 \\
$\%$ & & & $100 \%$ \\
Medianos & 139 & 120 & \\
N & $53,7 \%$ & $46,3 \%$ & 259 \\
$\%$ & & & $100 \%$ \\
Pequeños & 303 & 425 & \\
N & $41,6 \%$ & $58,4 \%$ & $100 \%$ \\
$\%$ & & & 1001 \\
Total & 454 & 547 & $100 \%$ \\
N & $45,4 \%$ & $54,6 \%$ & \\
$\%$ & & & \\
\hline
\end{tabular}

en la categoría de pequeños productores, estas diferencias resultaron ser estadísticamente significativas $\chi_{(2)}^{2}=21,124, \mathrm{p}<0,001$.

\section{Los grandes productores}

Tras la revisión de los artículos recogidos en la categoría de Psychology Educational de la WoS en el periodo estudiado, entre los investigadores que trabajan en instituciones españolas destaca la figura de Eduardo Vidal Abarca (Universidad de Valencia) con 12 trabajos. Con 10 trabajos destaca José Carlos Nuñez (Universidad de Oviedo) y con 8 trabajos cada uno, Emilio Sánchez (Universidad de Salamanca) y Antonio Valle (Universidad de A Coruña). Hay que mencionar que entre los 14 grandes productores, se encuentra un autor, Pedro Rosario, profesor de la Universidad de Minho (Portugal), que aunque no trabaja en una institución española, sin embargo destaca dentro de la producción científica española en esta área por su coautoría en varios artículos publicados con investigadores españoles.

En el análisis de las revistas donde han publicado sus artículos los grandes productores, si tenemos en cuenta el cuartil donde se encuentran en la base de datos JCR (tabla 2), se observa que 10 de los autores han publicado alguno de sus trabajos en revistas clasificadas en el cuartil 1. El número total de artículos en los que han colaborado los autores con mayor producción es de 101, los cuales se distribuyen en 20 firmas en revistas clasificadas en el cuartil 1 de la base de datos JCR (19,8\%), 29 firmas (28,7\%) en revistas clasificadas en el cuartil 2, 12 firmas (11,9\%) en revistas de cuartil 3 y 40 firmas (39,6\%) en revistas incluidas en el cuartil 4.

\section{Análisis de las redes de colaboración científica}

La colaboración en los trabajos científicos es un tema que ha sido objeto de numerosos estudios, principalmente por sus conexiones con los grupos de investigación. El índice firmas/trabajo se ha ido convirtiendo en un indicador estadístico de la literatura científica tan interesante como sencillo. En el análisis de la colaboración científica es importante verificar cuántas firmas presenta cada trabajo. Cuando se analiza la colaboración científica en los

Tabla 2

Detalle de la producción científica de los grandes productores de artículo en la categoría Psychology Educational de la Web of Science (2004-2013)

\begin{tabular}{|c|c|c|c|c|c|}
\hline \multirow[t]{2}{*}{ Autor / centro de trabajo } & \multirow[t]{2}{*}{ Revistas donde han publicado } & \multicolumn{4}{|c|}{$\mathrm{N}^{\circ}$. artículos y cuartil año de la publicación } \\
\hline & & Q1 & Q2 & Q3 & Q4 \\
\hline Eduardo Vidal Abarca (UVEG) & $\begin{array}{l}\text { Contemporany Educational Psychology; Discourse Processes; Infancia y } \\
\text { Aprendizaje; Journal of Educational Psychology; Learning and Individual } \\
\text { Differences; Learning and Instructions }\end{array}$ & 5 & 2 & & 5 \\
\hline José Carlos Nuñez (UO) & $\begin{array}{l}\text { European Journal of Psychology of Education; Infancia y Aprendizaje; } \\
\text { Learning and Individual Differences; Metacognition and Learning; Revista } \\
\text { de Psicodidáctica }\end{array}$ & 1 & 3 & 2 & 4 \\
\hline Emilio Sánchez (US) & $\begin{array}{l}\text { European Journal of Psychology of Education; Infancia y Aprendizaje; } \\
\text { Instructional Science; Learning and Instruction; Reading and Writing }\end{array}$ & 2 & 1 & 1 & 4 \\
\hline Antonio Valle (UAC) & $\begin{array}{l}\text { European Journal of Psychology of Education; Learning and Individual } \\
\text { Differences; Metacognition and Learning; Psychology in the Schools; } \\
\text { Revista de Psicodidáctica }\end{array}$ & & 3 & 2 & 3 \\
\hline Francisco. Cano (UG) & $\begin{array}{l}\text { British Journal of Educational Psychology; Educational and Psychological } \\
\text { Measurement; European Journal of Psychology of Education; Learning and } \\
\text { Instruction }\end{array}$ & 2 & 1 & 2 & 2 \\
\hline Juan Ignacio Pozo (UAM) & $\begin{array}{l}\text { British Journal of Educational Psychology; Cognition and Instruction; } \\
\text { Infancia y Aprendizaje; Learning and Instruction; Psychology of Music; } \\
\text { Revista de Psicodidáctica }\end{array}$ & 1 & 4 & & 2 \\
\hline Raquel Cerdán (UVEG) & $\begin{array}{l}\text { Infancia y Aprendizaje; Journal of Educational Psychology; Learning and } \\
\text { Individual Differences; Learning and Instruction; Revista de Psicodidáctica }\end{array}$ & 2 & 2 & & 2 \\
\hline Laura Gil (UVEG) & $\begin{array}{l}\text { Contemporary Educational Psychology; Infancia y Aprendizaje; Journal } \\
\text { of Educational Psychology; Learning and Instruction; Reading Research } \\
\text { Quarterly }\end{array}$ & 4 & & & 3 \\
\hline Jesús Alonso Tapia (UAM) & $\begin{array}{l}\text { Infancia y Aprendizaje; Learning and Individual Differences; Learning } \\
\text { and Instruction }\end{array}$ & 1 & 1 & & 4 \\
\hline Gualberto Buela Casal (UG) & Learning and Individual Differences; Revista de Psicodidáctica & 1 & 3 & 1 & 1 \\
\hline Cándido J. Inglés (UMH) & $\begin{array}{l}\text { Infancia y Aprendizaje; Learning and Individual Differences; Measurement } \\
\text { and Evaluation in Counseling and Development; Psychology in the } \\
\text { Schools; Revista de Psicodidáctica }\end{array}$ & & 3 & 1 & 2 \\
\hline José M. García Fernández (UA) & $\begin{array}{l}\text { Infancia y Aprendizaje; Learning and Individual Differences; Measurement } \\
\text { and Evaluation in Counseling and Development; Psychology in the } \\
\text { Schools; Revista de Psicodidáctica }\end{array}$ & & 3 & 1 & 2 \\
\hline Carles Monereo (UAB) & $\begin{array}{l}\text { School Psychology International; Infancia y Aprendizaje; Learning } \\
\text { and Instruction; Revista de Psicodidáctica }\end{array}$ & 1 & 1 & 1 & 3 \\
\hline Pedro Rosario (UM) & $\begin{array}{l}\text { European Journal of Psychology of Education; Metacognition } \\
\text { and Learning; Revista de Psicodidáctica }\end{array}$ & & 2 & 1 & 3 \\
\hline
\end{tabular}

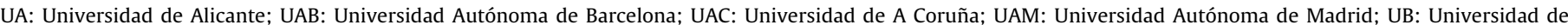

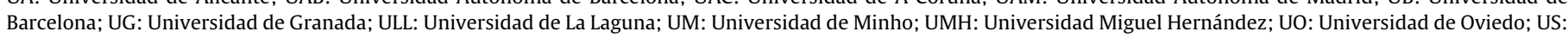
Universidad de Salamanca; UVEG: Universidad de Valencia. 

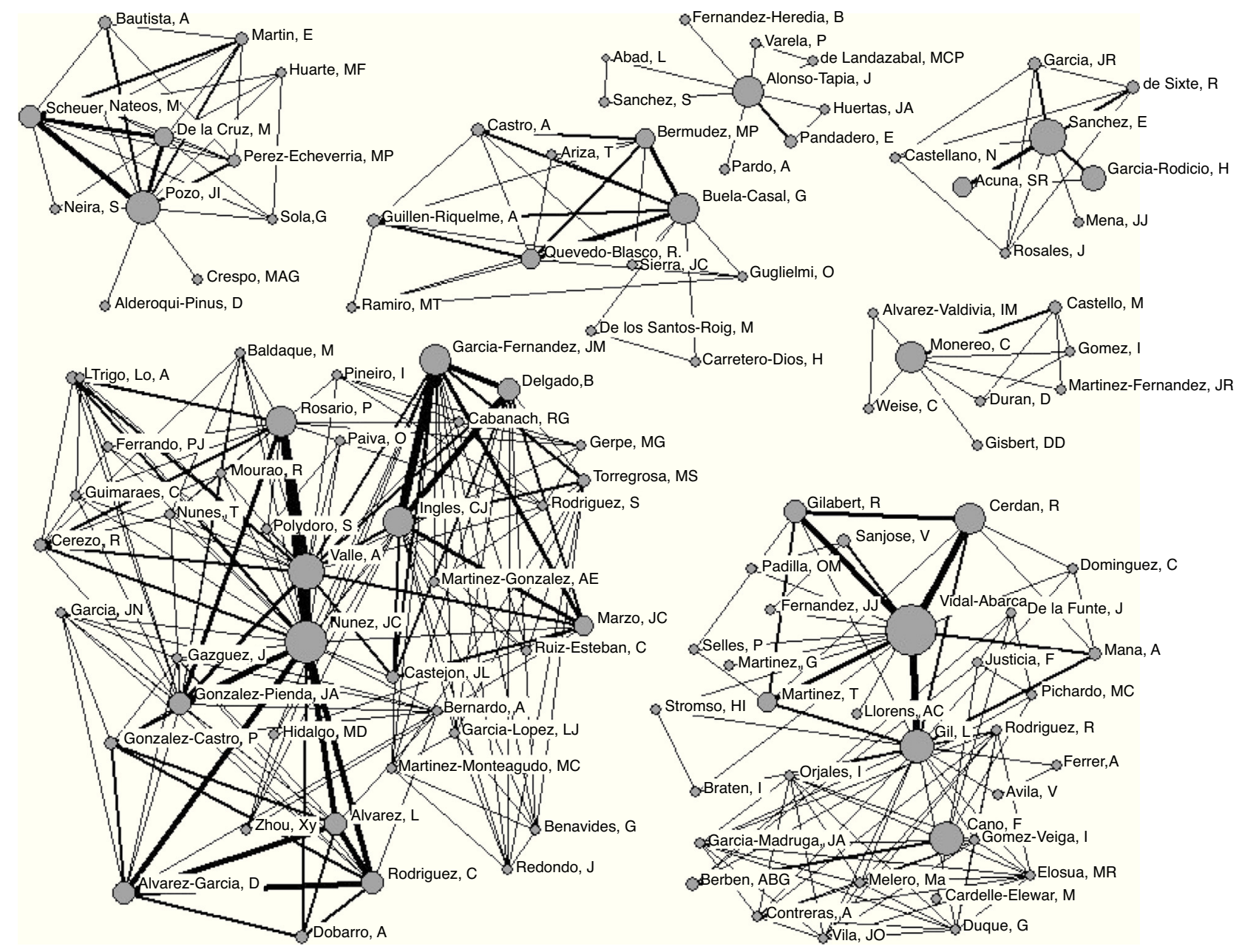

Figura 2. Red de los grandes productores y todos sus colaboradores de artículos en la categoría Psychology Educational de la Web of Science (2004-2013).

trabajos españoles incluido en la categoría Psychology Educational de la WoS durante el período 2004-2013, se observa que predominan los trabajos en colaboración frente a los trabajos realizados individualmente. El 92,3\% de los trabajos (421) están firmados por dos o más autores, y solamente el 7,7\% de los trabajos (35) están firmados por un solo autor. Los trabajos publicados en colaboración se mueven en un rango que va desde los 124 trabajos con dos autores por trabajo hasta un trabajo firmado por 10 autores distintos.

Para la identificación de clusters o agrupaciones de autores aplicando las técnicas de análisis de redes sociales se han identificado todas las combinaciones de pares de autores presentes en cada uno de los trabajos, es decir, las coautorías. El término cluster se refiere al conjunto de nodos (en nuestro caso, autores) altamente conectados entre sí mediante enlaces (relaciones de coautoría). El umbral o la intensidad de colaboración es el valor utilizado para formar las agrupaciones de los autores y hace referencia a la frecuencia de coautoría entre las parejas de autores. Refleja las relaciones entre los autores a la hora de publicar los resultados de sus investigaciones de forma conjunta y se utiliza en los estudios bibliométricos como criterio para determinar las agrupaciones identificadas como grupos de investigación.

Dada la imposibilidad de representar gráficamente todas las relaciones existentes que surgen al aplicar diferentes relaciones de colaboración, para representar la red de todos los autores que han sido objeto de estudio en nuestro trabajo, se ha decidido representar la red de los autores con mayor producción. Se identificaron
8 redes de actividad en la que se distribuyen un total 119 autores (fig. 2). Tal como se ha mencionado anteriormente, los nodos representan a los autores y el tamaño de los nodos es directamente proporcional a su productividad y el grosor de los vínculos, también es directamente proporcional a la cantidad de trabajos en colaboración de los dos autores a los que conecta.

En este período estudiado, el grupo más números está compuesto por 39 autores, entre los que se encuentran cinco de los grandes productores, José Carlos Nuñez (Universidad de Oviedo), Antonio Valle (Universidad de A Coruña), Cándido J. Inglés (Universidad Miguel Hernández), José M. García Fernández (Universidad de Alicante) y Pedro Rosario (Universidad de Minho). El segundo núcleo más numeroso está formado por 26 investigadores, entre los que se encuentran el mayor productor de trabajos de Psicología Educativa durante el período estudiado, Eduardo Vidal Abarca (Universidad de Valencia), junto con otras dos grandes productoras, Raquel Cerdán (Universidad de Valencia) y Laura Gil (Universidad de Valencia), todos ellos de la Universidad de Valencia.

Tal como se observa en la figura 2, los seis grupos restantes de investigadores tienen un menor número de miembros. El tercer grupo más numeroso es el de Juan Ignacio Pozo (Universidad Autónoma de Madrid), con 12 investigadores y el cuarto, el de Gualberto Buela-Casal (Universidad de Granada), con 11 investigadores. Los restantes grupos son el de Jesús Alonso Tapia (Universidad Autónoma de Madrid), con 9 investigadores, y el de Emilio Sánchez (Universidad de Salamanca) y el de Carles Monereo (Universidad Autónoma de Barcelona) con ocho investigadores cada uno de ellos 

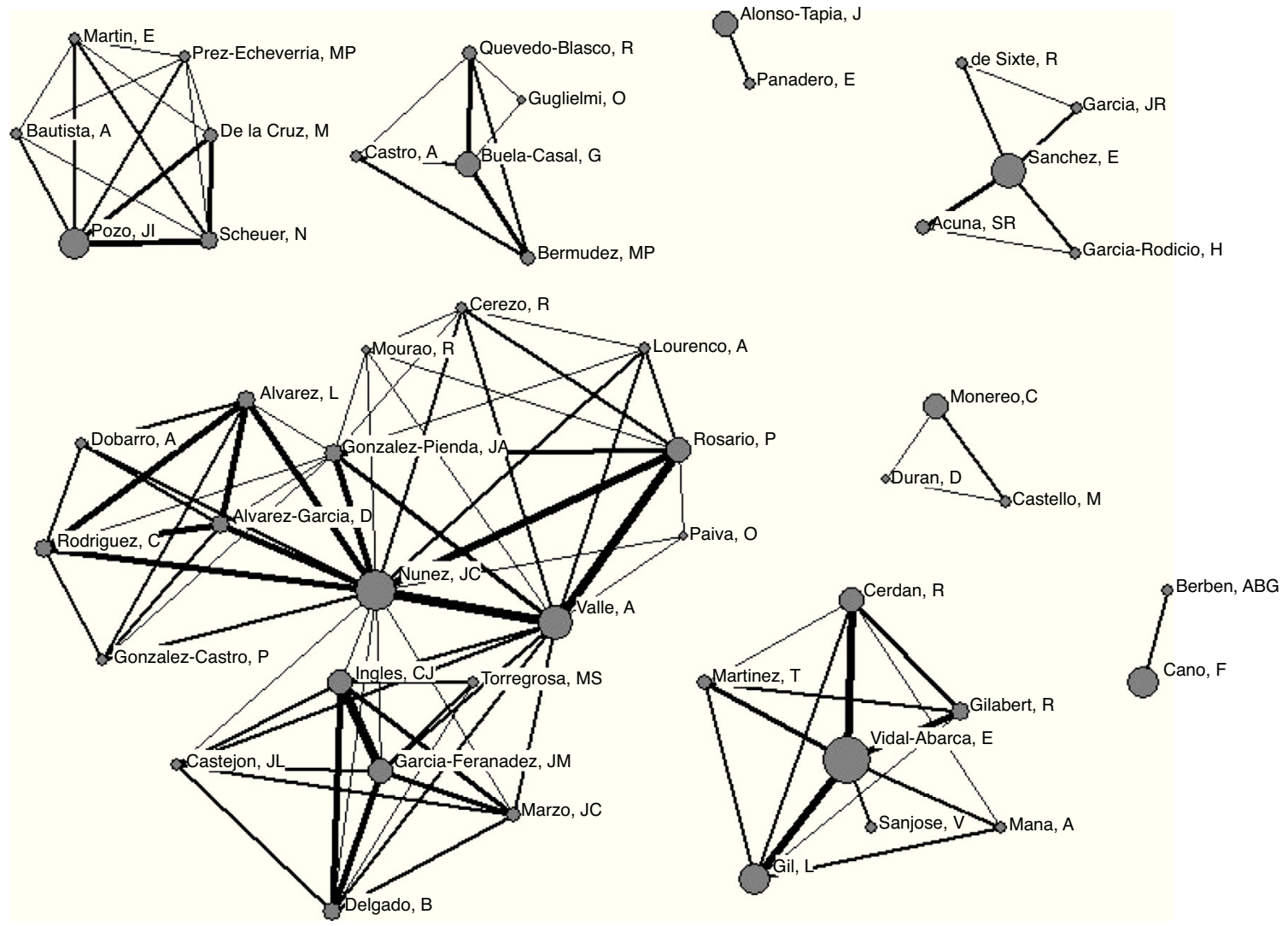

Figura 3. Red de los grandes productores y colaboradores en dos o más artículos en la categoría Psychology Educational de la Web of Science (2004-2013).

y el grupo de Francisco Cano (Universidad de Granada), con 6 investigadores.

Con el fin de visualizar mejor la red de los grandes productores $\mathrm{y}$, conocer el peso que tienen los investigadores eventuales dentro de los diferentes grupos de investigación, se ha representado a los grandes productores junto con aquellos investigadores con los que han colaborado en dos o más trabajos (fig. 3). En este caso son un total de 49 autores los que se distribuyen entre las ocho redes de actividad, siendo 70 (58,8\%) los autores eventuales que forman parte de la red de grandes productores. En este caso, se puede ver que el tamaño de los grupos se ha reducido ostensiblemente. Los grupos de mayor tamaño están compuestos por 19 investigadores (entre los que se encuentran cinco de los grandes productores) y 7 investigadores (tres de ellos grandes productores). En tercer lugar, hay un grupo formado por 6 investigadores, dos grupos formado por 5 investigadores, uno formado por 3 investigadores y 2 formados por dos investigadores cada uno de ellos. En esta figura se puede ver con mayor claridad el papel de intermediación que tienen algunos investigadores dentro de su propio grupo, como es el caso de Eduardo Vidal Abarca, Emilio Sánchez, José Carlos Núñez y Antonio Valle, que además de grandes productores, tienen un papel relevante dentro de su grupo de investigación.

\section{Análisis de las instituciones}

La totalidad de los artículos estudiados fueron firmados por autores pertenecientes a 179 instituciones diferentes entre universidades, centros de investigación, hospitales, centros educativos entre otros. Se observa que el sector institucional que tiene una mayor producción son las universidades, al que pertenecen 951 autores, 95\% del total, mientras que 50 autores firman los artículos perteneciendo a instituciones diferentes al ámbito universitario. Las universidades con un mayor número de autores y de firmas son la Universidad del País Vasco, seguida por la de Valencia, la de Barcelona y la Autónoma de Madrid. Son 11 universidades a las que están adscritos aproximadamente la mitad de los autores de artículos recogidos en la categoría Psychology Educational de la WoS en el periodo estudiado, en concreto 508 autores lo que representa el $50,7 \%$ del total de autores tal y como se puede observar en la tabla 3. Por lo que respecta a la presencia de grandes, medianos y eventuales productores resaltar que de entre las instituciones con mayor número de autores y de firmas por trabajo, 5 de ellas presentan algún gran productor, destacando la Universidad de Valencia con 3 grandes productores. Destacar que la Universidad del País Vasco es la que presenta un mayor número de autores, 70 en total, sin embargo no cuenta con ningún gran productor, y a su vez es la institución que cuenta con un mayor porcentaje de medianos productores.

\section{Las revistas}

Los 456 artículos se publicaron en 34 revistas pertenecientes a cinco países, siendo el número total de revistas incluidas en la categoría de Psychological Educational del JCR durante el período analizado de 54. El mayor número de artículos firmados por autores pertenecientes a alguna institución española fueron publicados en dos revistas, Infancia y Aprendizaje (35,5\%) y Revista de Psicodidáctica (19,1\%), ambas españolas (tabla 4), sumando un total de 249 trabajos. Esto supone que el 54,6\% de la producción total de artículos es publicada en revistas españolas. Con respecto a las revistas extranjeras, las más utilizadas fueron European Journal of Psychology of Education, con 36 trabajos (8\%) y Learning and Individual Differences, con 27 trabajos (6\%). Tras España, el resto de países de edición de las revistas por orden de trabajos publicados son Estados Unidos ( 75 artículos en 20 revistas), Inglaterra (50 artículos en 7 revistas), 
Tabla 3

Universidades con mayor producción en revistas incluidas en la categoría Psychology Educational de la Web of Science en el periodo $2004-2013$

\begin{tabular}{|c|c|c|c|c|c|c|c|c|}
\hline \multirow[t]{2}{*}{ Universidades } & \multirow[t]{2}{*}{ N. ${ }^{\circ}$ autores (n. firmas) } & \multirow[t]{2}{*}{$\%^{*}$} & \multicolumn{2}{|c|}{ Eventuales } & \multicolumn{2}{|c|}{ Medianos } & \multicolumn{2}{|c|}{ Grandes } \\
\hline & & & $\mathrm{N}$ & $\%$ & $\mathrm{~N}$ & $\%$ & $\mathrm{~N}$ & $\%$ \\
\hline U. País Vasco & $70(96)$ & 7,0 & 47 & 13,4 & 23 & 15,5 & 0 & 0 \\
\hline U. Valencia & $62(110)$ & 6,2 & 47 & 13,4 & 12 & 8,1 & 3 & 33,4 \\
\hline U. Barcelona & $57(86)$ & 5,7 & 40 & 11,4 & 17 & 11,9 & 0 & 0 \\
\hline U. Autónoma de Madrid & $55(88)$ & 5,4 & 38 & 10,8 & 15 & 10 & 2 & 22,2 \\
\hline U. Granada & $51(90)$ & 5,1 & 29 & 8,2 & 20 & 13,5 & 2 & 22,2 \\
\hline U. Autónoma de Barcelona & $39(61)$ & 3,9 & 27 & 7,7 & 11 & 7,4 & 1 & 11,1 \\
\hline U. Oviedo & $39(77)$ & 3,9 & 23 & 6,6 & 15 & 10 & 1 & 11,1 \\
\hline U. Málaga & $37(44)$ & 3,7 & 31 & 8,8 & 6 & 4,1 & 0 & 0 \\
\hline U. Sevilla & $37(54)$ & 3,7 & 25 & 7,1 & 12 & 8,1 & 0 & 0 \\
\hline U. de La Laguna & $32(49)$ & 3,2 & 23 & 6,6 & 9 & 6,0 & 0 & 0 \\
\hline U. Murcia & $29(45)$ & 2,9 & 21 & 6,0 & 8 & 5,4 & 0 & 0 \\
\hline Total & $508(800)$ & 50,7 & 351 & 100 & 148 & 100 & 9 & 100 \\
\hline
\end{tabular}

U: Universidad.

* \% de autores que firman artículos sobre el número total de autores.

Tabla 4

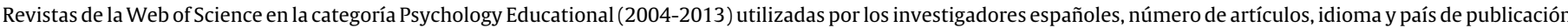
de la revista

\begin{tabular}{|c|c|c|c|c|}
\hline Título de la revista & $\mathrm{N} .^{\circ}$ artículos & $\%$ & Idioma & País de edición \\
\hline British Journal of Educational Psychology & 8 & 1,8 & Inglés & Inglaterra \\
\hline Child Development & 4 & 0,9 & Inglés & EE. UU. \\
\hline Cognition and Instruction & 1 & 0,2 & Inglés & EE. UU. \\
\hline Contemporary Educational Psychology & 4 & 0,9 & Inglés & EE. UU. \\
\hline Creativity Research Journal & 6 & 1,3 & Inglés & EE. UU. \\
\hline Discourse Processes & 4 & 0,9 & Inglés & EE. UU. \\
\hline Dyslexia & 3 & 0,7 & Inglés & Inglaterra \\
\hline Early Education and Development & 2 & 0,4 & Inglés & EE. UU. \\
\hline Educational and Psychological Measurement & 18 & 3,9 & Inglés & EE. UU. \\
\hline Educational Psychology & 7 & 1,5 & Inglés & Inglaterra \\
\hline European Journal of Psychology of Education & 36 & 8,0 & Inglés & Portugal \\
\hline Gifted Child Quarterly & 1 & 0,2 & Inglés & EE. UU. \\
\hline Infancia y Aprendizaje & 162 & 35,5 & Español & España \\
\hline Instructional Science & 5 & 1,1 & Inglés & Países Bajos \\
\hline Journal of Applied Research in Intellectual Disabilities & 5 & 1,1 & Inglés & Inglaterra \\
\hline Journal of Counseling Psychology & 2 & 0,4 & Inglés & EE. UU. \\
\hline Journal of Creative Behavior & 1 & 0,2 & Inglés & EE. UU. \\
\hline Journal of Educational Psychology & 3 & 0,7 & Inglés & EE. UU. \\
\hline Journal of Experimental Education & 7 & 1,5 & Inglés & EE. UU. \\
\hline Journal of Psychoeducational Assessment & 5 & 1,1 & Inglés & EE. UU. \\
\hline Journal of Research in Reading & 3 & 0,7 & Inglés & EE. UU. \\
\hline Journal of School Psychology & 1 & 0,2 & Inglés & Inglaterra \\
\hline Learning and Individual Differences & 27 & 6,0 & Inglés & Países Bajos \\
\hline Learning and Instruction & 14 & 3,1 & Inglés & Inglaterra \\
\hline Measurement and Evaluation in Counseling and Development & 1 & 0,2 & Inglés & EE. UU. \\
\hline Metacognition and Learning & 1 & 0,2 & Inglés & EE. UU. \\
\hline Psychology in the Schools & 6 & 1,3 & Inglés & EE. UU. \\
\hline Psychology of Music & 2 & 0,4 & Inglés & EE. UU. \\
\hline Reading and Writing & 12 & 2,6 & Inglés & Países Bajos \\
\hline Reading Research Quarterly & 3 & 0,7 & Inglés & EE. UU. \\
\hline Revista de Psicodidáctica & 87 & 19,1 & Español & España \\
\hline School Psychology International & 12 & 2,6 & Inglés & Inglaterra \\
\hline School Psychology Quarterly & 1 & 0,2 & Inglés & EE. UU. \\
\hline Social Psychology of Education & 2 & 0,4 & Inglés & Países Bajos \\
\hline
\end{tabular}

Países Bajos (46 artículos en 4 revistas) y Portugal (36 artículos en una revista).

\section{Conclusiones}

La producción española en Psicología Educativa recogida por la base de datos WoS se caracterizó en el periodo 2004 a 2013 por un aumento de la misma a lo largo de los años, en especial en los últimos cuatro años del periodo estudiado, siendo el año 2010 donde se dio una mayor producción, tendencia ya apuntada en cuanto al incremento de la producción de la ciencia española por la Fundación Española para la Ciencia y la Tecnología (2013). El número de trabajos recogidos en la WoS por miembros de alguna institución española fue de 509, siendo 1.120 el número de autores de los trabajos. Los artículos fueron la tipología documental más frecuente. De las 54 revistas que recoge la WoS en la categoría temática Psychology Educational del JCR los investigadores españoles han publicado en 34 de ellas, editadas en cinco países, Estados Unidos, Inglaterra, España, Países Bajos y Portugal, no habiendo publicado en revistas de Alemania, Japón, Rusia y Suiza, lo cual puede ser debido a la dificultad que entraña el idioma de la revista, si bien cabría valorar también el grado de colaboración científica de los investigadores españoles con investigadores de estos países, lo cual puede justificar la no publicación en estas revistas.

Gran parte de la producción de artículos se publicó en las revistas españolas, Infancia y Aprendizaje y la Revista de Psicodidáctica, que juntas suponen el $54,6 \%$ de toda la producción española durante el período estudiado, lo cual sugiere la importancia que ha tenido 
la inclusión de estas revistas en el JCR, circunstancia que puede estar detrás del aumento de la producción en los años venideros a la inclusión de las mismas y de la aparición de nuevos grupos de investigación. Por su parte, esta visibilidad a nivel internacional de los trabajos en Psicología Educativa realizados por investigadores que trabajan en instituciones españolas no solo es debido a la publicación en revistas españolas, sino también por la publicación, cerca de un $45,4 \%$ de trabajos, en revistas de otros países, principalmente de Estados Unidos e Inglaterra.

El número de investigadores que han firmado artículos científicos fue de 1.001, habiendo un mayor número de autoras, un 54,6\%, que de autores, un 45,4\%, si bien la producción fue mayor en el caso de los hombres, estando estos más representados en las categorías de medianos y grandes productores, resultados que están en consonancia con los trabajos de D'Amico et al. (2011), GonzálezAlcaide et al. (2010), Malouff et al. (2010), Maz-Machado et al. (2011), Olivas-Avila y Musi-Lechuga (2010) y entran en contradicción con los resultados de Olivas-Avila et al. (2012) donde no hubo diferencias en la producción de artículos en JCR en el área de Psicología Educativa en función del género entre profesores funcionarios en España. Llama la atención estos resultados si tenemos en cuenta que en España en el área de Psicología Evolutiva y de la Educación en el año 2011, el número de mujeres como personal docente investigador era mayor, representando el 62,7\% dentro de esta área de conocimiento, frente a un 37,3\% que son hombres (Instituto Nacional de Estadística, 2014), aspecto también señalado por Fong et al. (2009), si bien como señala Torres-Salinas et al. (2011), se ha de tener en cuenta la entrada reciente de la mujer en la universidad, lo cual puede estar detrás de una menor producción y de una menor presencia como grandes y medianas productoras.

Entre los grandes productores destacan las figuras de Eduardo Vidal Abarca perteneciente a la Universidad de Valencia con 12 trabajos, José Carlos Nuñez de la Universidad de Oviedo con 10 trabajos, y de Emilio Sánchez de la Universidad de Salamanca y Antonio Valle de la Universidad de A Coruña con 8 trabajos cada uno de ellos. Los 14 grandes productores presentan 121 firmas en diferentes trabajos, principalmente en revistas de cuartil 4 , si bien un $48,5 \%$ de las firmas se reparten en revistas de cuartil 1 y cuartil 2 , circunstancia que resalta la calidad de estos trabajos.

Los centros de trabajo más productivos en cuanto a artículos científicos son las universidades, en concreto a 11 de ellas pertenecen aproximadamente la mitad de los autores y de las firmas, destacando la Universidad del País Vasco con 70 autores, si bien, esta universidad no presenta ningún gran productor, aspecto que puede cambiar en años venideros, ya que es la universidad con mayor número de medianos productores, lo cual puede guardar relación con el impulso que puede proporcionar tener una revista en JCR perteneciente a esa institución, como sería el caso de la Revista de Psicodidáctica. Otros centros que destacan en producción son la Universidad Autónoma de Madrid, la de Barcelona y la de Valencia, siendo esta última la que cuenta con un mayor número de grandes productores.

Entre las limitaciones que presenta este trabajo hay que reseñar dos principalmente. La primera es que el trabajo se ha centrado en la publicación de artículos recogidos en la categoría de Psychology Educational de la base de datos WoS, esto constituye solo una parte de la producción científica española en esta área, ya que no se tienen en cuenta los trabajos publicados en revistas de otras áreas de la WoS como Education \& Educational Research; Psychology Multidisciplinary, Psychology Developmental o Education Special entre otras. A este respecto sería interesante realizar estudios donde se incluyese los trabajos publicados en estas áreas, ofreciendo así una imagen más real de la Psicología Educativa española.

La segunda limitación del trabajo obedece a los errores que pueden darse a la hora de incluir la totalidad de los artículos en la WoS, pues no todos los artículos publicados en las revistas de la WoS están incluidos en la propia base de datos, tal es el caso del artículo "A longitudinal assessment of the effectiveness of a school-based mentoring program in middle school», publicado en la revista Contemporary Educational Psychology, 38(1) en 2013, cuyos autores son J. Núñez, P. Rosario, G. Vallejo y J. A. González-Pienda, el cual no está recogido en la base de datos de la WoS.

También hay que tener en cuenta que las publicaciones constituyen solamente una faceta de la investigación científica y que la literatura científica es solamente una parte del sistema mucho más amplio y complejo que constituye la ciencia. Sin embargo, la publicación de los resultados de la investigación en revistas científicas es uno de los principios básicos en los que se fundamenta el sistema científico, por lo que este trabajo constituye una aproximación para conocer el estado de la producción científica española de la Psicología Educativa.

\section{Conflicto de intereses}

Los autores declaran no tener ningún conflicto de intereses.

\section{Referencias bibliográficas}

Barrios, M., Villarroya, A. y Borrego, A. (2013). Scientific production in psychology: a gender analysis. Scientometrics, 95, 15-23.

Beamonte, E., Casino, A. y Veres, E. J. (2013). Ranking global del 2010 de las universidades públicas españolas. Aula Abierta, 41, 75-86.

Bermúdez, M. P., Guillén-Riquelme, A., Gómez-García, A., Quevedo-Blasco, R., Sierra, J.C. y Buela-Casal, G. (2011). Análisis del rendimiento en el doctorado en función del sexo. Educación XXI, 14(1), 17-33.

Buela-Casal, G. (2010). Índices de impacto de las revistas científicas e indicadores para medir el rendimiento de los investigadores. Revista de Psicodidáctica, 15 3-19.

Buela-Casal, G., Bermúdez, M. P., Sierra, J. C., Quevedo-Blasco, R., Guillén-Riquelme, A. y Castro, A. (2013). Productividad y eficiencia en investigación por Comunidades Autónomas españolas según la financiación (2011). Aula Abierta, 41(2), 87-98.

Cunningham, P. (1997). The evaluation of European progmmes and the future of scientometrics. Scientometrics, 38, 71-85.

D’Amico, R., Vermigli, P. y Canetto, S. S. (2011). Publication productivity and career advancement by female and male psychology faculty: the case of Italy. Journal of Diversity in Higher Education, 4, 175-184 [consultado 15 Mar 2015]. Disponible en: http://psycnet.apa.org/doi/10.1037/a0022570.

Delgado-López-Cózar, E., Torres-Salinas, D., Jiménez-Contreras, E. y Ruíz-Pérez, R. (2006). Análisis bibliométrico y de redes sociales aplicado a las tesis bibliométricas defendidas en España (1976-2002): temas, escuelas científicas y redes académicas. Revista Española de Documentación Científica, 29, 493-524.

De Moya, F., Chinchilla, Z., Benavent-Pérez, M., Corera-Álvarez, E., González-Molina, A. y Vargas-Quesada, B. (2010). Indicadores bibliométricos de la actividad científica española. Madrid: Fecyt.

Evans, J., Hsieh, P. y Robinson, D. (2005). Women‘s involvement in Educational Psychology Journals from 1976 to 2004. Educational Psychology Review, 17, 263-271

Fong, C., Yoo, J., Jones, S., Torres, L. y Decker, M. (2009). Trends in female authorships, editorial board memberships, and editorships in Educational Psychology journals from 2003 to 2008. Educational Psychological Review, 21, 267-277.

Fundación Española para la Ciencia y la Tecnología (2013). (2013). Indicadores de actividad científica española 2010. Madrid: Fecyt.

Garfield, E. (2006). The history and meaning of the journal impact factor. JAMA, 295, 90-93.

González-Alcaide, G., Castelló-Cogollos, L., Bolaños-Pizarro, M., Alonso-Arroyo, A., Valderrama-Zurián, J. C. y Aleixandre-Benavent, R. (2010). Veinte años de investigación de la psicología española en Psicothema (1989-2008). Psicothema, 22, 41-50.

González-Sala, F., Fonseca-Baeza, S. y Osca-Lluch, J. (2014). La presencia española en comités de revistas iberoamericanas de psicología del Journal Citation Reports (2012). Revista Iberoamericana de Psicología y Salud, 5(2), 151-165.

Hirsch, J. E. y Buela-Casal, G. (2014). The meaning of the h-index. International Journal of Clinical and Health Psychology, 14, 161-164.

Instituto Nacional de Estadística (2014). Personal docente de los centros propios de las Universidades Públicas por Área de conocimiento, sexo y categoría. Estadística de la Enseñanza Universitaria en España. Curso 2010-2011 [consultado 15 Mar 2015]. Disponible en: http://www.ine.es/jaxi/tabla.do

Lotka, A. J. (1926). The frequency distribution of scientific productivity. Journal of the Washington Academy of Sciences, 16, 317-324.

Maz-Machado, A., Gutiérrez-Arenas, M. P., Bracho-López, R., Jiménez Franjul, N., Adamuz-Povedano, N. y Torralbo, R. (2011). Producción científica en Ciencias Sociales de las mujeres en Andalucía (2003-2007). Aula Abierta, 39, 63-72.

Malouff, J., Schutte, N. y Priest, J. (2010). Publication rates of Australian academic psychologists. Australian Psychologist, 45, 78-83. 
Mauleón, E., Hillán, L., Moreno, L., Gómez, I. y Bordons, M. (2013). Assessing gender balance among journal authors and editorial board members. Scientometrics, 95, 87-114.

Olivas-Avila, J. A. y Musi-Lechuga, B. (2010). Análisis de la producción de los profesores funcionarios de psicología en España en artículos de revistas de la Web of Science. Psicothema, 22, 909-916.

Olivas-Avila, J. A., Musi-Lechuga, B., Guillen-Riquelme, A. y Castro, A. (2012). Diferencias en la producción investigadora en tesis y artículos de los profesores funcionarios de Psicología en España en función del sexo. Anales de Psicología, 28, 597-603.

Osca-Lluch, J. (2011). La aportación de la mujer a la historia de la ciencia y de la técnica en España. Valencia: IHMCLP.

Osca-Lluch, J., Haba, J., Fonseca, S., Civera, C. y Tortosa, F. (2013). Tesis españolas sobre análisis bibliométrico en Psicología. Aula Abierta, 41(2), 99-110.
Sierra, J. C., Buela-Casal, G., Bermúdez, M. P. y Santos-Iglesias, P. (2009). Diferencias por sexo en los criterios y estándares de productividad científica y docente en profesores funcionarios en España. Psicothema, 21, 124-132.

Torres-Salinas, D., Muñoz-Muñoz, A. M. y Jiménez-Contreras, E. (2011). Análisis bibliométrico de la situación de las mujeres investigadoras de ciencias sociales y Jurídicas en España. Revista Española de Documentación Científica, 34 $11-28$

Velasco, B., Eiros, J. M., Pinilla, J. M. y San Román, J. A. (2012). La utilización de indicadores bibliométricos para evaluar la actividad investigadora. Aula Abierta, 40(2), 75-84.

Velasco, J., Vilariño, M., Amado, B. G. y Fariña, F. (2014). Análisis bibliométrico de la investigación española en psicología desde una perspectiva de género. Revista Iberoamericana de Psicología y Salud, 5, 105-118. 